\title{
OBESIDADE: CAUSAS E CONSEQUÊNCIAS EM CRIANÇAS E ADOLESCENTES
}

\author{
Flavia Hernandes, Centro Universitário de Maringá - CESUMAR - Maringá, Paraná - \\ Brasil \\ Meire Pereira Valentini, Ubiversidade Estadual de Maringá - UEM - Maringá, Paraná - \\ Brasil
}

\section{RESUMO}

A obesidade é definida como um distúrbio nutricional e metabólico caracterizado pelo aumento de massa adiposa no organismo, refletindo em um aumento de peso corpóreo. Um dos métodos mais utilizados para indicar riscos relacionados ao excesso de peso corporal é o Índice de Massa Corporal (IMC) e quando se trata de crianças e adolescentes, deve-se levar em conta a faixa etária analisada. O trabalho se caracteriza como pesquisa bibliográfica no qual foi realizado um levantamento de material de estudo, tendo como objetivo estudar a prevalência de sobrepeso e obesidade em crianças e adolescentes. Estudos demonstram que a atividade física é um mecanismo eficiente que contribui na redução e manutenção do peso corporal, aliada a outros hábitos positivos à saúde. Assim, conclui-se que devido ao grande índice de obesidade na atualidade, recomenda-se a intervenção através da prática da atividade física como projeto de vida familiar, com a finalidade de estimular e conscientizar sobre a importância e os benefícios que a mesma proporciona.

Palavras-Chave: Obesidade, Criança, Adolescente, Atividade física.

\section{OBESITY: A CONCERN EACH DAY MORE COMMON FOR CHILDREN AND TEENAGERS}

\begin{abstract}
Obesity is defined as a nutritional and metabolic disorder characterized by increased fat mass in the body, reflecting an increase in body weight. One of the methods used to indicate the risks related to excess body weight is the Body Mass Index (BMI), and when it comes to children and adolescents should take account of the age. The work is characterized as a literature in which a survey was conducted to study materials, in order to study the prevalence of overweight and obesity in children and adolescents. Studies show that physical activity is an efficient mechanism that contributes to the reduction and maintenance of body weight, combined with other positive health habits. Thus, it is concluded that due to the high rate of obesity nowadays, it is recommended to physical activity intervention and family life project, in order to stimulate and raise awareness of the importance and the benefits it provides.
\end{abstract}

KEY-WORDS: Obesity, Children, Teenager, Physical activity. 


\section{INTRODUÇÃO}

\section{Descrição do Problema}

Para Ramos e Barros Filho, ${ }^{1}$ a obesidade é um distúrbio nutricional e metabólico caracterizado pelo aumento da massa adiposa do organismo, que se refere no aumento do peso corpóreo. O aumento excessivo de gordura corporal pode provir do aumento do número de celular adiposas (hiperplasia) e/ou o tamanho das células adiposas (hipertrofia) conforme explicam Powers e Howley. ${ }^{2}$ Ainda sobre a visão de Ramos e Barros Filho ${ }^{1}$ a obesidade é uma condição clínica séria e prevalente, podendo se tornar o principal problema de saúde do século XXI e a primeira causa de doenças crônicas do mundo.

Tendo em vista que a obesidade é entendida como uma doença, a mesma raramente age sozinha, agrava muitos outros riscos como a hipertensão, doenças pulmonares, artrite, gota, toxemia na gravidez, problemas psicológicos, baixa tolerância a calor, função e tamanho do coração dentre outros fatores podendo influenciar de forma negativa na condição de saúde do individuo (POWERS e HOWLEY, 2005).

Para compreender melhor a abrangência do problema, Simão ${ }^{3}$ diz que cerca de trinta e sete milhões de norte-americanos apresentam $20 \%$ ou mais de peso acima do que é considerado saudável. De acordo com o mesmo autor, a obesidade atualmente é tão prejudicial à saúde que chega a ser comparada ao tabagismo, lipídios elevados no sangue e hipertensão quanto ao risco coronariano.

De acordo com a Organização Mundial de Saúde, ${ }^{4}$ atualmente, uma em cada dez crianças em todo o mundo é obesa, o que representa cerca de cento e cinqüenta e cinco milhões. No Brasil, pesquisa da Associação Brasileira para Estudos de Obesidade ${ }^{3}$ (ABESO), mostra que a obesidade infantil triplicou nos últimos vinte anos. Atualmente, quase $15 \%$ das crianças estão acima do peso e $5 \%$ são obesas.

Os índices de obesidade na infância e na adolescência têm aumentado de forma significativa. Conforme dados apresentados acima, patologias que antes eram 
diagnosticadas apenas na população adulta tem sido observadas e diagnosticadas freqüentemente em idades mais precoce.

A obesidade precoce tem como conseqüência a possibilidade de sua continuidade na idade adulta, e acarreta em um aumento dos riscos à saúde e das taxas de morbimortalidade. Diante desse contexto, levanta-se a seguinte questão: Quais as complicações provindas da obesidade na infância e na adolescência e as possibilidades de intervenções com a atividade física?

\section{OBJETIVOS}

\section{Objetivo Geral}

Estudar as causas e conseqüências da obesidade e do sobrepeso em crianças e adolescentes.

\section{Objetivos Específicos}

- Definir o que é sobrepeso e obesidade;

- Diagnosticar as causas do sobrepeso e obesidade na infância e na adolescência;

- Delimitar os principais métodos utilizados para diagnosticar a obesidade e sobrepeso;

- Descrever a importância da atividade física para a prevenção e o controle da obesidade.

\section{JUSTIFICATIVA}

A escolha desse tema deu-se através da vivência realizada no período de estágio supervisionado no qual pôde ser observado o alto índice de crianças e adolescentes que se encontravam aparentemente acima do peso. Durante o estágio pode ser verificado através de conversas com crianças e adolescentes que os maiores motivos que contribuem para o sobrepeso e obesidade provem de uma alimentação inadequada e a falta de atividade física, motivos nos quais me levam a questionar sobre a qualidade de vida destes indivíduos em um futuro próximo. 


\section{METODOLOGIA}

A pesquisa caracteriza-se como bibliográfica pois de acordo com Martins $\mathrm{Jr}^{6}{ }^{6}$ neste tipo de pesquisa, o pesquisador busca em fontes impressas e eletrônicas, da literatura as informações que necessita para desenvolver uma determinada teoria.

\section{REVISÃO DE LITERARUTA}

\section{Diagnóstico da Obesidade}

A obesidade é definida como um distúrbio nutricional e metabólico caracterizado pelo aumento de massa adiposa no organismo, refletindo em um aumento de peso corpóreo. ${ }^{1}$ Conforme Simão ${ }^{3}$, existe um padrão para o excesso de gordura aceito por pesquisadores. Este padrão é estabelecido em $20 \%$ de gordura para homens e $30 \%$ de gordura para mulheres. Essas estimativas foram definidas a partir da relação do IMC e a morbimortalidade, o que não pode ser facilmente aplicado a crianças uma vez sendo que as complicações da obesidade infanto-juvenil são manifestadas com maior freqüência na vida adulta. $^{7}$

Pollock e Wilmore ${ }^{8}$ entendem o sobrepeso e a obesidade como distúrbios crônicos complexos, relacionados a vários fatores que desequilibram o balanço energético na direção do ganho de peso. De acordo com os autores, ambos os termos são usados como se fossem sinônimos, porém, no ponto de vista técnico possuem significados diferentes. Sobrepeso é o peso corporal que excede do peso normal ou padrão dos indivíduos da mesma raça e sexo baseado na altura, idade e constituição física. Já a obesidade é definida pela OMS, ${ }^{4}$ como a "doença na qual a gordura se acumulou no organismo a tal ponto que a saúde pode ser afetada".

Para diagnosticar se a criança e/ou o adolescente se encontra em estado de obesidade ou sobrepeso, não basta simplesmente observá-los e compará-los com o padrão de boa aparência. $\mathrm{O}$ excesso de peso pode muito facilmente ser confundido com obesidade, contudo existem diferenças significativas entre ambos. Bouchard ${ }^{9}$ (2003) sustenta: o sobrepeso é sob vários aspectos, muito diferente da obesidade, na qual a obesidade 
caracteriza-se por um excesso significativamente maior de peso e, de massa de tecido adiposo do que o sobrepeso; entretanto a situação é ainda mais complexa.

Conforme Powers e Howley, ${ }^{2}$ existem diversos meios para se diagnosticar a obesidade como: Diluição de Isótopos, Absorção de Fótons, Potássio-40, Pesagem Hidrostática, Absortometria Radiológica de Dupla Energia, Interactância de Raios Infravermelhos, Radiografia, Ultra-Sonografia, Ressonância Magnética, Condutividade Elétrica Total, Bioimpedância Elétrica e Espessura das Dobras Cutâneas.

Ainda que seja possível encontrar na literatura diversas formas de aferir a massa corporal do indivíduo, o método mais utilizado e de baixo custo é o Índice de Massa Corporal (IMC). Este recurso é o mais utilizado não só por sua fácil execução, mas também por ser aceito pela comunidade científica. Para Powers e Howley, ${ }^{2}$ o IMC é o resultado do seguinte cálculo: peso do indivíduo dividido pela sua altura ao quadrado $\left(\mathrm{kg} / \mathrm{m}^{2}\right)$.

De acordo com a OMS, ${ }^{10}$ o IMC de crianças e adolescentes está relacionado à faixa etária, conforme representado nas tabelas 1 e 2.

Tabela 1: Linha de corte do IMC para indivíduos do sexo masculino entre 5 e 19 anos.

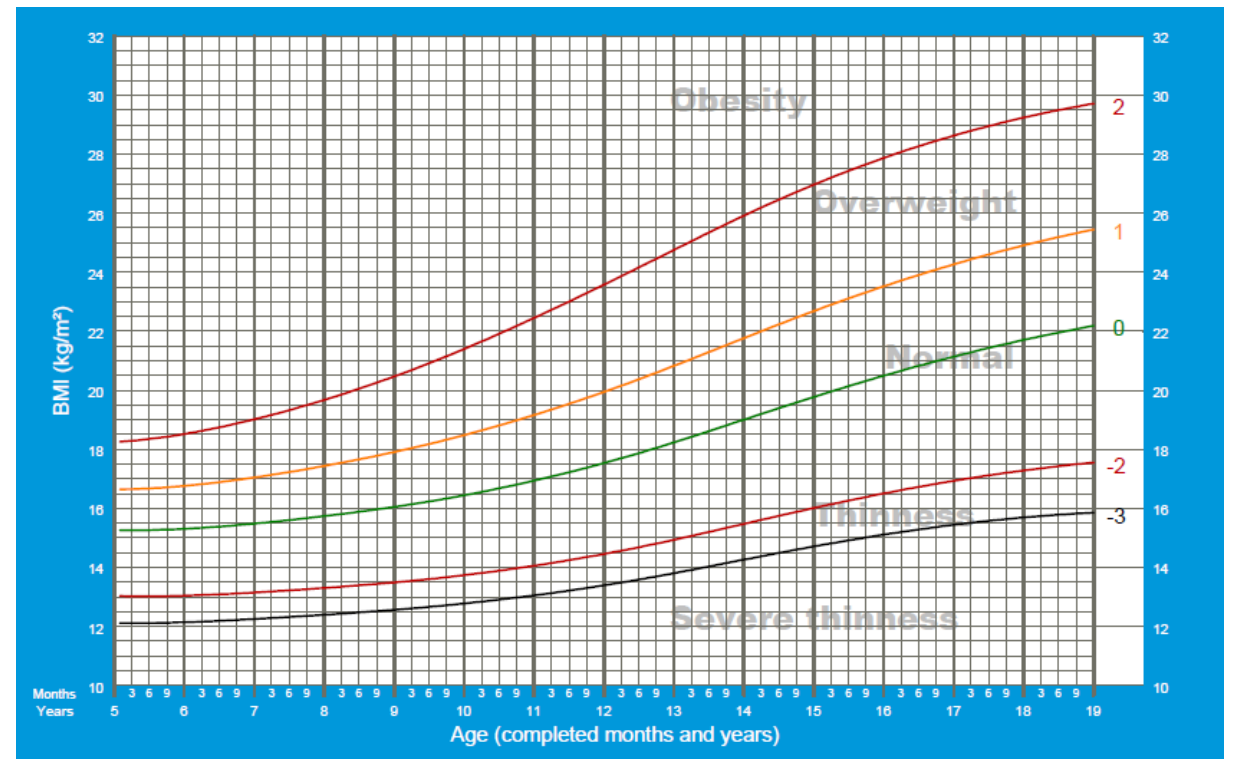

Fonte: ${ }^{10}$. 
Tabela 2: Linha de corte do IMC para indivíduos do sexo feminino entre 5 e 19 anos.

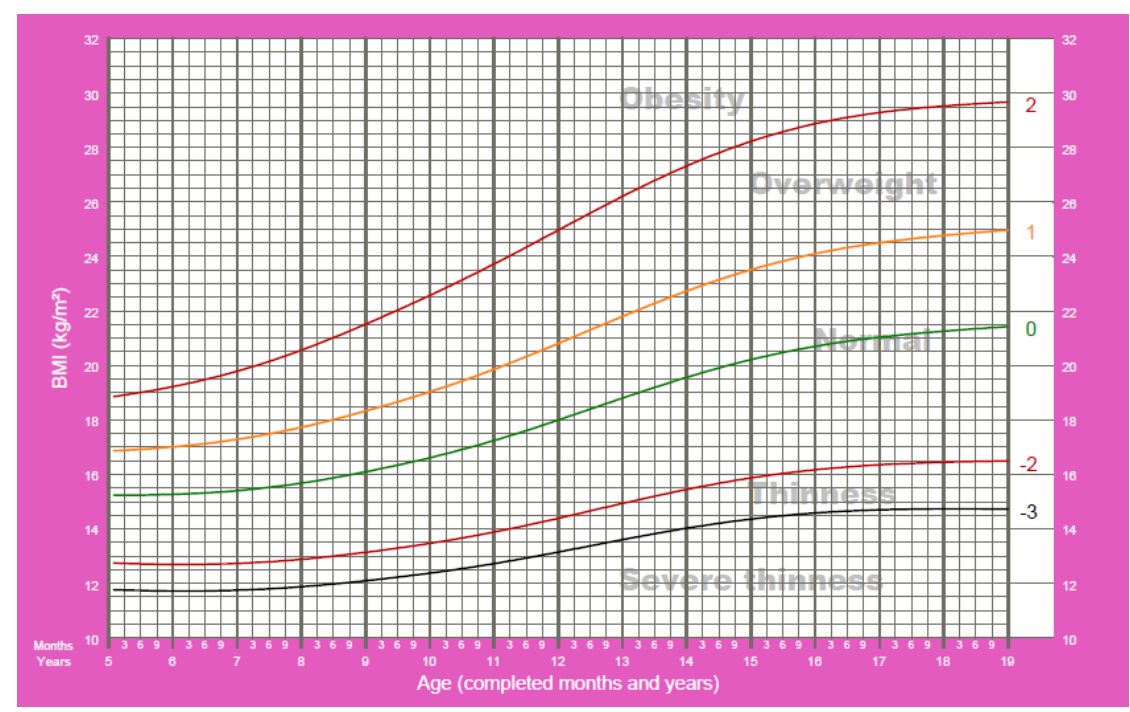

Fonte: ${ }^{10}$

De acordo com a OMS ${ }^{10}$ deve-se realizar o calculo do IMC da criança e/ou adolescente e comparar o valor com a idade e o sexo apresentados nas tabelas anteriores. Dado o ponto de encontro dos mesmos, deve ser verificado o escore no qual o indivíduo se encontra.

O quadro abaixo descreve a classificação dos valores de IMC para crianças e adolescentes:

Quadro 1: Escores de IMC por idade em crianças de 5 a 19 anos.

\begin{tabular}{|c|c|}
\hline IMC por Idade - 5 a 19 an & \\
\hline VALORES CRITICOS & DIAGNOSTICO NUTRICIONAL \\
\hline$<$ Escore-z -3 & Magreza acentuada \\
\hline $\begin{array}{c}\geq \text { Escore-z }-3 \text { e }< \\
\text { Escore-z-2 }\end{array}$ & Magreza \\
\hline $\begin{array}{c}\geq \text { Escore-z }-2 \mathrm{e} \leq \\
\text { Escore-z }+1\end{array}$ & Eutrofia \\
\hline $\begin{array}{c}\geq \text { Escore-z+1 e } \leq \\
\text { Escore-z }+2\end{array}$ & Sobrepeso \\
\hline $\begin{array}{c}\geq \text { Escore-z }+2 \text { e } \leq \\
\text { Escore-z }+3\end{array}$ & Obesidade \\
\hline$>$ Escore- $z+3$ & Obesidade grave \\
\hline
\end{tabular}

Fonte: ${ }^{10}$

Conexões: revista da Faculdade de Educação Física da UNICAMP, Campinas, v. 8, n. 3, p. 47-63, set./dez. 2010. ISSN: 1983-9030 
A importância de saber como a gordura está distribuída, se deve ao fato de que dependendo de onde a gordura está mais localizada o indivíduo tem mais ou menos chances de adquirir certas doenças e tem aumentada ou diminuída a probabilidade de mortalidade. Segundo Dionne e Tremblay, ${ }^{11}$ os tipos de fenótipos da obesidade sob uma perspectiva anatômica são: tipo I que é caracterizada pelo excesso de massa corporal ou porcentagem de gordura distribuída por todo corpo; tipo II que se constitui a forma andróide, caracterizada pelo acúmulo de gordura do tronco, particularmente no abdômen.

As crianças obesas, normalmente, apresentam gordura tipo I, ou seja, a gordura não está centralizada em determinado lugar do corpo. Com a finalidade de se entender melhor a totalização da gordura, Viuniski, ${ }^{12}$ lembra: nas crianças menores encontramos mais frequentemente a obesidade generalizada, sem um nítido predomínio de distribuição da adiposidade. À medida que vão crescendo, o tipo de obesidade é melhor definido.

Alem da distribuição de gordura no tecido adiposo, é importante compreender se a obesidade ocorre de forma hipertrófica, hiperplástica ou de ambos. Conforme Powers e Howley, ${ }^{2}$ quando uma pessoa é submetida a uma restrição dietética, o tamanho das células adiposas diminuem, porém o seu número continua em igual proporção. De acordo com os mesmos autores (IBID) tanto a atividade física quanto a intervenção dietética em crianças e jovens obesos podem diminuir a velocidade de aumento da quantidade de células adiposas, relacionada à incapacidade das mesmas em eliminarem sua obesidade quando adultas.

\section{Etiologia da Obesidade}

As mudanças demográficas e econômicas, associadas ao desenvolvimento científico de nossa época, propiciaram o aumento da expectativa de vida da população, controle das doenças infecto-contagiosas contribuindo para a mudança no perfil alimentar e de morbimortalidade dos países do ocidente. ${ }^{13}$ Neste contexto, as doenças crônicodegenerativas e o alto consumo de alimentos processados industrialmente - ricos em gorduras saturadas, açúcares e altamente calóricos - têm assumido um aumento do número de casos de obesidade e doenças relacionadas. ${ }^{14}$ 
O desenvolvimento que se inicia no nascimento e se estende até o óbito, pode ser definido como um processo em que ocorrem mudanças contínuas e graduais do organismo humano, de um nível mais simples para um mais complexo. A ordem natural desse desenvolvimento pode ser modificada pela falta de vivências de jovens adeptos do sedentarismo. ${ }^{15}$

Levando em consideração que o estilo de vida começa a ser formado na infância, é possível afirmar que crianças com baixo nível de atividade motora, por exemplo, podem tornar-se adultos sedentários e, conseqüentemente não terem um bom índice de qualidade de vida. ${ }^{16}$ Isso sugere que informações relacionadas com as variáveis do aspecto morfológico (crescimento e composição corporal), variações de desempenho motor e estágio maturacional podem nos dar indicadores dos níveis de saúde dos indivíduos mais jovens. ${ }^{16-17}$

Junto à comunidade científica já existe um consenso quanto ao entendimento das causas da obesidade, pois admite-se que esta é uma doença multifatorial e seu desenvolvimento pode ser determinado por um ou pela soma de fatores como genético, ambiental, comportamental e sócio-cultural. ${ }^{7,18-19}$

De acordo com Bray, ${ }^{20}$ o excesso de peso se tornou um malefício frequente na vida contemporânea, uma vez que as pessoas estão muito mais sedentárias e consumindo uma grande quantidade de alimentos ricos em gordura. Neste sentido o autor afirma: o peso corporal aumentado está associado ao risco de mortalidade também aumentado.

Além do excesso de ingestão alimentar e do sedentarismo, existem implicações genéticas no controle do peso corporal. Conforme Leite, ${ }^{7}$ a programação e controle genético são capazes de produzir a manutenção fisiológica de uma determinada quantidade de gordura para cada indivíduo. O genótipo pode influenciar a forma de distribuição de gordura, a taxa metabólica de repouso, a habilidade de utilizar a energia dos alimentos, a composição corporal, a atividade do tecido adiposo, a lipólise e o comportamento na alimentação. $^{7}$

Agras et. al. ${ }^{21}$ afirma que a obesidade dos pais é apontada como o maior fator de risco para o desenvolvimento de obesidade em crianças. Para isso Viuniski, ${ }^{12}$ elucida que Conexões: revista da Faculdade de Educação Física da UNICAMP, Campinas, v. 8, n. 3, p. 47-63, set./dez. 2010. ISSN: 1983-9030 
estudos clássicos em famílias de obesos revelaram que se ambos os pais forem obesos, 2/3 dos filhos serão obesos. Se somente um dos pais for obeso, o risco de obesidade nas suas crianças cai para $50 \%$. Se ambos os pais forem pessoas magras, as suas crianças terão um risco de menos de $10 \%$ para obesidade.

Fatores de grande influência nas causas do sobrepeso da criança são atribuídos ao aumento no consumo de produtos ricos em gorduras com alto valor calórico, diminuição da prática de exercícios físicos, tempo de televisão diária e avanços tecnológicos da sociedade moderna. A prática de assistir à televisão durante várias horas por dia, os jogos eletrônicos, o abandono da amamentação são fatores que devem ser considerados na determinação do crescimento da obesidade infantil. ${ }^{22}$ Assim observa-se que o aumento no número de crianças obesas está relacionado à mudança no estilo de vida e aos hábitos alimentares.

Segundo Leão et al., ${ }^{23}$ o Brasil já é apontado entre os países com rápida elevação do sobrepeso e obesidade - quando avaliada a partir do IMC - principalmente em crianças e adolescentes, mesmo em populações mais carentes.

Segundo Taddei, ${ }^{22}$ é extremamente necessário identificar as causas da obesidade de cada indivíduo, porém descobrir o efeito desencadeador da obesidade se tornou algo muito difícil. A alteração no peso de um indivíduo pode não ter apenas uma causa e sim várias e normalmente fatores internos e externos estão em constante interação. De acordo com Mello, Luft e Meyer ${ }^{24}$ os fatores internos são: necessidades e características psicológicas, imagem corporal, valores e experiências pessoais, auto-estima, preferências alimentares, saúde e desenvolvimento psicológico. Quanto aos fatores externos, estes compreendem em: unidade familiar e suas características, atitudes de pais e amigos, valores sociais e culturais, mídia, alimentos rápidos, conhecimentos de nutrição e manias alimentares.

\section{Complicações da Obesidade}

Recentemente, estudos procuram abranger levantamentos populacionais, na tentativa de fornecer subsídios na análise da prevalência do sobrepeso e da obesidade em todas as idades e camadas sociais. Esses estudos, além de descrever o fenômeno, podem contribuir para a monitoração do nível de saúde e qualidade de vida da população. ${ }^{18}$

Conexões: revista da Faculdade de Educação Física da UNICAMP, Campinas, v. 8, n. 3, p. 47-63, set./dez. 2010. ISSN: 1983-9030 
Para Ramos e Barros Filho, ${ }^{1}$ o estado de obesidade e de sobrepeso é uma condição clínica séria e prevalente, podendo se tornar o principal problema de saúde do século XXI e a primeira causa de doenças crônicas do mundo.

De acordo com a Organização Mundial de Saúde, ${ }^{4}$ atualmente uma em cada dez crianças em todo o mundo é obesa, o que representa cerca de cento e cinqüenta e cinco milhões de pessoas. No Brasil, uma pesquisa da Associação Brasileira para Estudos de Obesidade, ${ }^{5}$ mostra que a obesidade infantil triplicou nos últimos vinte anos. Atualmente, quase $15 \%$ das crianças estão acima do peso e $5 \%$ são obesas.

A obesidade na infância e na adolescência tem aumentado de forma progressiva. Conforme dados apresentados acima, patologias que antes eram diagnosticadas apenas na população adulta têm sido observadas e diagnosticadas frequentemente em idades mais precoces.

Várias complicações podem ocorrer na saúde de uma criança com sobrepeso, além disso, uma criança obesa aumenta a probabilidade de se tornar um adulto obeso, o que pode gerar problemas de saúde tendo como consequência até mesmo a morte. Sob esta visão Escrivão e Lopez, ${ }^{14}$ informam que a maioria das complicações da obesidade iniciadas na infância e na adolescência acaba se manifestando na fase adulta levando ao aumento da morbimortalidade e à diminuição da esperança de vida.

Tendo em vista que a obesidade hoje é considerada como uma doença, a mesma raramente age sozinha, agravando então muitos outros riscos como a hipertensão, doenças pulmonares, artrite, gota, toxemia na gravidez, problemas psicológicos, baixa tolerância a calor, função e tamanho do coração dentre outros demais fatores podendo então influenciar de forma negativa na condição de saúde do individuo. ${ }^{2}$

Conforme Bray, ${ }^{20}$ as condições físicas, sociais e psicológicas associadas com a obesidade são: osteoartrite, diabete mellito não insulino dependente, hiperlipidemia, doença cardíaca, acidente vascular cerebral, hipertensão, alguns tipos de câncer, doença da vesícula biliar, gota, distúrbios alimentares, distúrbios do sono e distúrbios de humor. 
Para Suplicy, ${ }^{25}$ outro aspecto que é desencadeado pelo surgimento da obesidade nas pessoas com pouca idade é o fator psicossocial. As crianças obesas têm sua auto-estima bastante diminuída, sentem-se rejeitadas e tendem a isolar-se de modo que esses pequenos cidadãos apresentam grande dificuldade de interação na escola. Este pensamento reflete as consequências psicossociais da obesidade que derivam de valores ligados à cultura atual que considera o corpo gordo como feio e pouco saudável.

Pode-se verificar que diversos autores têm um ponto de vista paralelo quando se diz respeito às doenças que estão associadas com a obesidade.

Estudos transversais com escolares em todo país têm se tornado uma prática comum, na tentativa de identificar e descrever a prevalência do sobrepeso e obesidade em crianças e adolescentes, já que a obesidade infanto-juvenil tem estreita ligação com a obesidade do adulto. $^{26}$

O aumento da obesidade e suas seqüelas como problema clínico e social é que tem estimulado estudos que conforme Ramos e Barros Filho, ${ }^{1}$ nos levam à percepção do quanto o sobrepeso e a obesidade estão cada vez mais presentes em todas as comunidades, atingindo todas as idades.

Para compreender melhor a abrangência do problema, encontram-se dados de que cerca de 37 milhões de norte-americanos apresentam $20 \%$ ou mais de peso acima do que é considerado saudável. A obesidade atualmente é tão prejudicial à saúde que chega ser comparada ao tabagismo, lipídios elevados no sangue e hipertensão quanto ao risco coronariano. $^{3}$

Verifica-se então o tamanho da abrangência de problemas causados pela obesidade e sobrepeso, e ainda, como os mesmos têm influenciado de forma negativa as perspectivas do país e ainda mais, do mundo. 


\title{
O papel da atividade física na prevenção e no controle da obesidade
}

Para evitar que a prevalência da obesidade continue crescendo, surge a necessidade de adotarem-se medidas de prevenção. Estas podem ser por meio do aumento do gasto calórico pelo exercício ou pela diminuição na ingestão calórica.

\begin{abstract}
A diminuição da quantidade de atividade física contribui de forma importante para o aumento do sobrepeso e da obesidade. Existem prováveis mecanismos através dos quais o exercício pode auxiliar na perda e manutenção do peso. Alguns deles seriam o aumento do gasto diário de energia, a redução do apetite, o aumento da taxa metabólica de repouso, o aumento da massa muscular, o aumento do efeito térmico de uma refeição, a elevação do consumo de oxigênio, a otimização dos índices de mobilização e utilização de gordura, bem como uma sensação de auto-suficiência e bem-estar. ${ }^{26}$
\end{abstract}

O exercício físico regular é de grande relevância na prevenção e tratamento tanto da obesidade como de várias outras doenças, como o diabetes e as doenças cardiovasculares e os benefícios podem ser adquiridos geralmente como consequências de melhoras cardiorrespiratórias e alterações na composição corporal (como diminuição de gordura corporal e/ou aumento de massa magra). ${ }^{28}$

Trabalhos enfatizam que o objetivo primordial de qualquer tratamento para obesidade deve estar centrado em melhoras do estilo de vida, e não simplesmente na perda de peso. Conforme Oded Bar-Or ${ }^{29}$ as crianças, ao contrário dos adultos, raramente fazem exercícios para a manutenção da saúde. Conforme o autor, elas precisam sentir - se gratificadas durante a realização das atividades.

De acordo com Souza e Virtuoso $\mathrm{Jr}^{30}$ os programas para controle de peso devem combinar com a restrição moderada do consumo energético em conjunto com a realização de exercícios específicos para a perda de gordura. Conforme Fernandez et al. ${ }^{31}$ os exercícios mais eficazes para a perda de gordura são os exercícios aeróbios e os exercícios contra resistência.

A recomendação atual para a saúde é que tanto adultos quanto crianças realizem no mínimo 30 minutos diários de atividade contínua ou acumulada, de intensidade leve a moderada, gerando então um déficit de 500 a $1000 \mathrm{Kcal}$ diárias chegando a perda de $1 \mathrm{Kg}$ de gordura por semana. ${ }^{4}$ 
Como se pode perceber este é um tema exaustivamente abordado na literatura, desde o final da década de 70 até os dias atuais. Apesar de haver ainda informações contraditórias quanto ao tipo de treinamento ideal, é consenso que os benefícios do treinamento físico regular sobre parâmetros metabólicos e a consequente redução na mortalidade são fundamentais na melhora da qualidade de vida das pessoas obesas. ${ }^{32}$

\section{CONCLUSÃO}

Conforme a literatura apresentada pôde-se compreender que a obesidade é definida como um distúrbio nutricional e metabólico no qual ocorre um aumento de tecido adiposo acarretando no aumento de peso. Dentre as diversas causas da obesidade, têm-se maior ênfase na alta ingestão alimentar e no baixo nível de atividade física. Um dos métodos mais utilizados para diagnosticar se o indivíduo é obeso é o Índice de Massa Corporal (IMC), entretanto quando se trata de crianças e adolescentes, deve-se levar em conta a faixa etária analisada.

Ao desenvolver um programa de atividade física para crianças e adolescentes, é necessário também trabalhar a motivação da criança para que a mesma se mantenha ativa tornando esta atividade um hábito de vida. O ideal seria que o incentivo à prática da atividade física fosse um projeto familiar no qual todos participassem, auxiliando assim na conscientização e no estímulo à prática da atividade física desde a infância do indivíduo.

É importante também saber o motivo que faz com que a criança e adolescentes optem por serem sedentárias, para então escolher um melhor método para ajudá-los. Sendo assim, profissionais da saúde e educadores deveriam incluir uma análise das atividades habituais da criança e as barreiras que devem ser superadas para que ela adote um estilo de vida mais ativo e mais saudável. 


\section{REFERÊNCIAS}

${ }^{1}$ RAMOS, A. M. P. P. e BARROS FILHO, A. A. "Prevalência da obesidade em adolescentes de Bragança Paulista e sua relação com a obesidade dos pais”. Arquivos Brasileiros Endocrinológico Metabólico, v. 6, p. 663-667, 2003.

${ }^{2}$ POWERS, S. K. e HOWLEY, E. T. Fisiologia do exercício: teoria e aplicação ao condicionamento e ao desempenho. 5. ed. Barueri: Manole, 2005.

${ }^{3}$ SIMÃO, R. Fisiologia e prescrição de exercícios para grupos especiais. 3. ed. Rio de Janeiro: Phorte, 2007.

4 ORGANIZAÇÃO Mundial da Saúde (OMS). Doenças crônico-degenerativas e obesidade: estratégia mundial sobre alimentação saudável, atividade física e saúde. 2003. Disponível em: http://www.opas.org.br/sistema/arquivos/d_cronic.pdf Acesso em: 29 jan. 2009

${ }^{5}$ ASSOCIAÇÃO Brasileira para Estudos da Obesidade (ABESO). 2009. O Programa escola saudável. Disponível em: http://www.abeso.org.br/revista/revista20/escola_saudavel.htm Acesso em: 24 fev. 2009.

${ }^{6}$ MARTINS JR., J. Como escrever trabalhos de conclusão de curso: instruções para planejar e montar, desenvolver, concluir, redigir e apresentar trabalhos monográficos e artigos. Petrópolis: Vozes, 2008.

${ }^{7}$ LEITE, N. Obesidade infanto-juvenil: Efeitos da atividade física e da orientação nutricional sobre a resistência insulínica. 2005. 162f. Monografia (Especialização em Saúde da Criança e do Adolescente) - Universidade Federal do Paraná,Curitiba, 2005.

${ }^{8}$ POLLOCK, M. L.; WILMORE, J. H. Exercício na saúde e na doença: avaliação e prescrição para prevenção e reabilitação. 2. ed. Rio de Janeiro: Medsi, 1993.

${ }^{9}$ BOUCHARD, C. Atividade física e obesidade. São Paulo: Manole, 2003. 
${ }^{10}$ ORGANIZAÇÃO Mundial da Saúde (OMS). IMC por idade (5 a 19 anos). 2007. Disponível em: http://www.who.int/growthref/who2007_bmi_for_age/en/index.html. Acesso em: 03 nov. 2009.

${ }^{11}$ DIONNE, I.; TREMBLAY, A. Balanço energético e de nutrientes em humanos. In: BOUCHARD, C. (Org.). Atividade física e obesidade. São Paulo: Manole, 2003. p.173205.

${ }^{12}$ VIUNISKI, N. Obesidade infantil: um guia prático. Rio de Janeiro: EPUB, 2000.

${ }^{13}$ MINAYO, M. C. S. Os Muitos brasis: saúde e população na década de 80. São Paulo: Rio de Janeiro: Hucitec-Abrasco, 1995.

${ }^{14}$ ESCRIVÃO, M. A. M. S.; LOPEZ, F. A. Prognóstico da obesidade na infância e na adolescência. In: FISBERG, M. (Org.). Obesidade infância e adolescência. São Paulo: Fundo editorial BYK, 1995. p. 146-155.

15 SORIANO, F. F. S.; MORTATTI, A. L. Análise da correlação entre dados morfológicos, desempenho motor e maturação em escolares de 11 a 14 anos de idade, no município de São Paulo (SP). Conscientiae Saúde, v.6, n. 1, p. 179-188, 2007.

16 GUEDES, D. P.; GUEDES, J. E. R. P. Crescimento, composição corporal e desempenho motor de crianças e adolescentes. São Paulo: CLR Balieiro, 1997.

17 FARIAS, E. S.; SALVADOR, M. R. D. Antropometria, composição corporal e atividade física de escolares. Revista Brasileira de Cineantropometria e Desenvolvimento Humano, v. 7, n. 1, p. 21-29, 2005.

${ }^{18}$ GUEDES, D. P e GUEDES, J. E. R. P. Controle de peso corporal: composição, atividade física e nutrição. Londrina: Midiograf, 1998.

${ }^{19}$ ESCRIVÃO, M. A. M. S. et al. Obesidade exógena na infância e na adolescência. Jornal de Pediatria, Rio de Janeiro, v. 76, p. 305-310, 2000. 
${ }^{20}$ BRAY, G. A. Sobrepeso, Mortalidade e Morbidade. In: Bouchard, C. (Org.). Atividade física e obesidade. São Paulo: Manole, 2003. p. 35-62.

${ }^{21}$ AGRAS, W. S. et. al. Risk factors for childhood owerweight: a prospective study from birth to 9,5 years. Journal of Pediatrics, v. 145, p. 20-25, 2004.

${ }^{22}$ TADDEI, J. A. A. C. Epidemiologia da obesidade na infância e adolescência. In: FISBERG, M. (Org.). Obesidade na infância e adolescência. São Paulo: Fundo Editorial BYK, 1995. p. 14-18.

${ }^{23}$ LEÃO, L. S. C. et al. Prevalência de obesidade em escolares de Salvador, BA. Arquivo Brasileiro de Endocrinologia e Metabologia, v. 47, n. 2, p.151-157, abr. 2003.

${ }^{24}$ MELLO, E. D.; LUFT, V. C.; MEYER, F. Obesidade infantil: como podemos ser eficazes? Jornal de Pediatria, v. 80, p. 173 - 181, 2004.

${ }^{25}$ SUPLICY, H. L. Quais as consequências do excesso de peso? as doenças associadas à obesidade. In: HALPERN, A.; MANCINI, M. C. (Org.). Manual de obesidade para o clínico. São Paulo: Roca, 2002. p. 61-84.

${ }^{26}$ CARVALHO, C. M. R. R. et al. Consumo alimentar de adolescentes matriculados em colégio particular de Teresina, Piauí, Br. Revista de Nutrição, Campinas, v.14, n.2, p.8593, maio/ago. 2001.

${ }^{27}$ HAUSER, C.; BENETTI, M.; REBELO, F. P. V. Estratégias para o emagrecimento. Revista de Cineantropometria e Desempenho Humano, v. 6, n. 1, p. 72-81, 2004.

28 FRANCISCHI, R. P.; PEREIRA, L. O.; LANCHA JR., A. H. Exercício, comportamento alimentar e obesidade: revisão dos efeitos sobre a composição corporal e parâmetros metabólicos. Revista Paulista de Educação Física, São Paulo, v. 15, n. 2, p. 117-146, julho/dezembro, 2001. 
${ }^{29}$ ODED BAR-OR, M. D. A Epidemia de obesidade juvenil: a atividade física é relevante? Gatorade Sports Science Institute, v. 16, n. 2, jul./set. 2003. Disponível em: http://www.gssi.com.br/. Acesso em: 08 set. 2009.

${ }^{30}$ SOUZA, L. M.; VIRTUOSO JR, J. S. A Efetividade de Programas de Exercício Físico no Controle do Peso Corporal. Revista Saúde.Com, Bahia, v. 1, p. 71-78, 2005.

${ }^{31}$ FERNANDEZ, A. C. et. al. Influência do treinamento aeróbio e anaeróbio na massa de gordura corporal de adolescentes obesos. Revista Brasileira de Medicina do Esporte. v. 10, n. 3, p. 152-158, maio/jun. 2004.

${ }^{32}$ VOTRUBA, S. B.; HORVITZ, M. A.; SCHOELLER, D. A. The role of exercise in the treatment of obesity. Nutrition, New York, v. 16, p.179-88, 2000. 